As $x$ decreases, $K_{\nu}(x)$ increases rapidly while $I_{\nu}(x)$ decreases rapidly. Therefore, the error introduced by the truncation, decreases in the case of $K_{\nu}(x)$, and increases in the case of $I_{\nu}(x)$. This difficulty is avoided by calculating $\rho$. That is, at $x=2$, e.g., where $K_{\nu}(x)$ is still accurately given by the solution of the differential equations, equations (17) and (18)

$$
\rho=\frac{\tilde{I}_{\nu}(x)-I_{\nu}(x)}{K_{\nu}(x)} .
$$

At $x=2, I_{\nu}(x)$ is easily obtained by standard methods, e.g. the defining series, or integral representation.

M. Goldstein

Los Alamos Scientific Laboratory,

R. M. Thaler

Los Alamos, New Mexico

1. W. E. Milne, "The numerical determination of characteristic numbers," Phys. Rev., v. 35,1930 , p. $863-867$.

2. HAROLD JEFFREYS, "On certain approximate solutions of linear differential equations of the second order," London Math. Soc., Proc., v. 23, 1923, p. 428-436.

The mathematical method first given by Jeffreys was independently derived in connection with an important physical problem by

a. G. WentzeL, "Eine Verallgemeinerung der Quantenbedingungen für die Zwecke der Wellenmechanik," Zeits. f. Physik, v. 38, 1926, p. 518-529.

b. H. A. Kramers, "Wellenmechanik und halbzahlige Quantisierung," Zeits. f. Physik, v. 39,1926, p. 828-840.

c. L. Brillouin, "La mécanique ondulatoire de Schrödinger; une méthode générale de résolution par approximations sucessives," Comptes Rendus, v. 183, 1926, p. 24-26.

d. L. Brillouin, "Remarques sur la mécanique ondulatoire," J. de Physique, v. 7, 1926, p. $353-368$, and is commonly referred to as the JWKB approximation.

\title{
Numerical Experiments in Potential Theory Using the Nehari Estimates
}

\author{
By U. W. Hochstrasser
}

1. Introduction. It is well known that the Dirichlet problem can be solved by using a set of harmonic functions which are orthogonal over the boundary [7]. Prior to the availability of high speed computers, however, the method was not frequently used since generating sets of orthogonal functions by hand computations is very laborious. With the advent of high speed computers interest in the method has been renewed and several papers have appeared recently $[1,2,3]$.

In using the method, the numerical analyst is particularly interested in estimates of the error committed by replacing infinite orthogonal expansions by finite ones. Nehari [1] recently has given such estimates for a number of Dirichlet problems. The purpose of the present paper is to report the results of some numerical experiments which were designed to investigate how close these estimates come to the actual errors in certain particular examples. An existing computer program [4] was used for the orthogonalization of a given set of vectors on the SEAC com-

Received 27 March 1957. The preparation of this paper was sponsored by the Office of Scientific Research of the Air Research and Development Command, United States Air Force, while the author was a guest worker at the National Bureau of Standards. 
puter at the National Bureau of Standards. Several boundary value problems, each involving the same irregular pentagon, were solved. For two of these problems the exact solution is known so that one can compare the exact errors with the estimates. In all cases the number of orthogonal harmonic functions used to approximate the solution was varied. The effect of using a sophisticated choice of the harmonic functions was also studied.

2. Dirichlet problems for an irregular pentagon. In the experiments an irregular pentagon inscribable in a circle was used in all the cases as the region on which the Dirichlet problem was defined (see Figure 1).

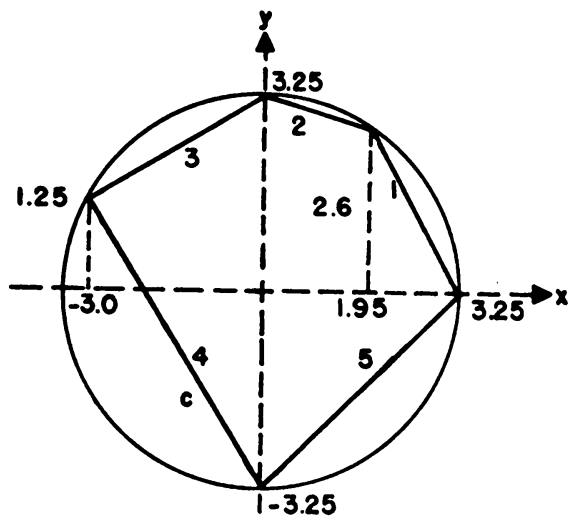

FIG. 1

For this region the following Dirichlet problems were considered:

(a) The boundary values are given by

$$
U(x, y)=\operatorname{Re}(\cos z)=\operatorname{Re} \cos (x+i y)=\cos x \cosh y .
$$

(b) The boundary values are given by

$$
U(x, y)=\operatorname{Re} \frac{1}{z+4}=\frac{x+4}{(x+4)^{2}+y^{2}} .
$$

(c) The boundary values are equal to one on one side and zero on all the others. (The solutions of these problems are the harmonic measures for the sides.)

The solution of the first Dirichlet problem changes sign in the interior and on the boundary of the region. In the second case the solution is always positive on the whole region. The set of functions which were used to approximate the solution in the cases $a$ and $b$ consisted of harmonic polynomials obtained by orthonormalizing $1, \operatorname{Re} z, \operatorname{Im} z, \operatorname{Re} z^{2}, \operatorname{Im} z^{2}, \ldots$ on the region. It was expected that with a few functions one would obtain a better approximation in the second case than in the first.

In order to find approximate values for the harmonic measures of the sides of the pentagon one can start from the harmonic measure of the circumscribed circle. Let the segment $2 \alpha$ be that part of the circumference of this circle which is cut out by the side of the pentagon with boundary values equal to 1 . Then the harmonic measure $u_{0}$ which has the value 1 on the segment $2 \alpha$ and 0 on the rest of the circumference 
is given by

(see Figure 2)

$$
u_{0}=\frac{\varphi-\alpha}{\pi-2 \alpha}
$$

$$
\begin{aligned}
& \varphi=\arccos \left[\frac{\left(x-x_{2}\right)\left(x-x_{1}\right)+\left(y-y_{2}\right)\left(y-y_{1}\right)}{\left.\sqrt{\left[\left(x-x_{1}\right)^{2}+\left(y-y_{1}\right)^{2}\right]\left[\left(x-x_{2}\right)^{2}+\left(y-y_{2}\right)^{2}\right]}\right]}\right. \\
& \alpha=\frac{1}{2} \arccos \left[\frac{x_{1} x_{2}+y_{1} y_{2}}{x_{1}{ }^{2}+y_{1}{ }^{2}}\right]
\end{aligned}
$$

where $\varphi$ is the angle belonging to any point in the region and $\left(x_{1}, y_{1}\right),\left(x_{2}, y_{2}\right)$ are the coordinates of the end points of that side of the pentagon with boundary values

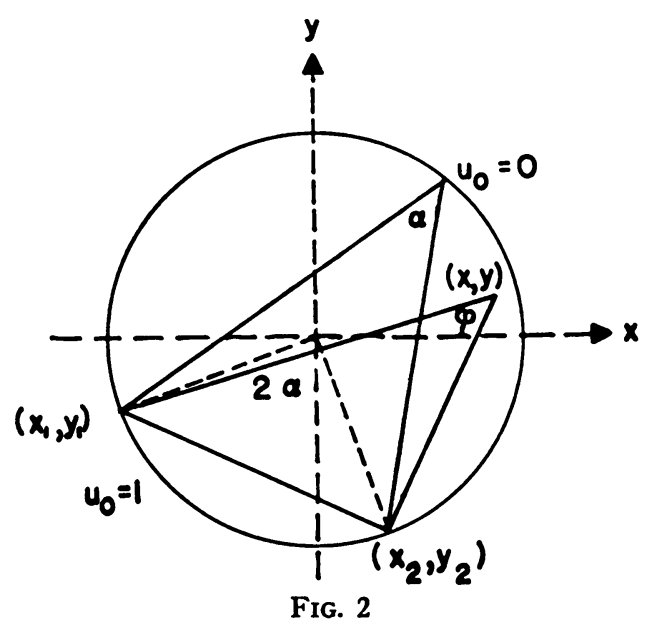

equal to 1 . In addition to these harmonic measures the harmonic polynomials 1 , $\operatorname{Re} z, \operatorname{Im} z, \operatorname{Re} z^{2}, \operatorname{Im} z^{2}, \cdots$ were used for obtaining the set of approximating functions. These sets were orthonormalized with respect to the inner product

$$
(f, g)=\int_{c} f(x, y) g(x, y) d s, \quad d s^{2}=d x^{2}+d y^{2}
$$

where the integration is along the boundary $c$ of the region.

If $n$ functions $1, \operatorname{Re} z, \operatorname{Im} z, \operatorname{Re}\left(z^{2}\right), \operatorname{Im}\left(z^{2}\right) \cdots$ are used as basic set one obtains after orthonormalization a system of $n$ orthonormal harmonic polynomials $u_{v}(x, y)$. The approximate solution of the Dirichlet problem is given then by the sum

$$
u(x, y)=\sum_{v=1}^{n}\left[\int_{c} U(X, Y) u_{v}(X, Y) d S\right] u_{v}(x, y),\left(d S^{2}=d X^{2}+d Y^{2}\right) .
$$

For the numerical computations the contour integrals appearing in these computations must be replaced by an integration formula involving finite sums. Since the contour consists of straight lines, splitting the integral into five integrals corresponding to the five sides of the contour was suggested.

For their evaluation an eight point Gaussian-type integration rule was chosen involving the end points of the interval of integration as given by Radau [5]. 
This rule is exact for polynomials up to degree 13. It corresponds therefore to a seven point ordinary Gaussian integration rule. Since it involves the endpoints, the computational work for the eight point Radau rule and the seven point Gaussian rule is, however, the same for a closed contour. Radau's formula has the added convenience of using the corner points of the pentagon. Thus the contour integral is replaced by a sum over 35 products of the form

$$
\int_{c} G(x, y) d s \sim \sum_{i=1}^{i=5} \sum_{j=1}^{j=7} w_{i j} G\left(A_{j} x_{i}+\left(1-A_{j}\right) x_{i-1}, \quad A_{j} y_{i}+\left(1-A_{j}\right) y_{i-1}\right)
$$

where $\left(x_{i}, y_{i}\right)$ are the coordinates of the vertices of the pentagon

$$
\left(x_{0}=x_{5}, y_{0}=y_{5}\right)
$$

and the weights $w_{i j}$ as well as the coefficients $A_{j}$ are given by the integration rule

$$
\begin{aligned}
& w_{i j}=\frac{1}{2} \sqrt{\left(x_{i}-x_{i-1}\right)^{2}+\left(y_{i}-y_{i-1}\right)^{2}} H_{j} \quad j=2,3, \ldots, 7, i=1,2, \ldots, 5 \\
& w_{i 1}=\frac{1}{2}\left\{\sqrt{\left(x_{i}-x_{i-1}\right)^{2}+\left(y_{i}-y_{i-1}\right)^{2}}\right. \\
& \left.+\sqrt{\left(x_{i-1}-x_{i-2}\right)^{2}+\left(y_{i-1}-y_{i-2}\right)^{2}}\right\} H_{1} . \quad\left(x_{-1}=x_{4}, y_{-1}=y_{4}\right) \\
& \begin{array}{llllllll}
j & 1 & 2 & 3 & 4 & 5 & 6 & 7
\end{array}
\end{aligned}
$$

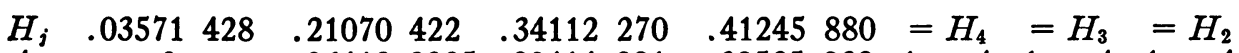

$$
\begin{aligned}
& \begin{array}{ccccccc}
A_{j} & 0 & .064129925 .20414991 & .39535039 & 1-A_{4} & 1-A_{3} & 1-A_{2}
\end{array}
\end{aligned}
$$

The orthonormalization is carried out by the Gram-Schmidt process: If $f_{i}$ is a set of $n$ vectors with $N$ components then a system of orthonormal vectors $u_{i}$ with components $u_{i k}$, is found by the formulas

$$
\begin{aligned}
& u_{1}=a_{11} f_{1} \\
& u_{2}=a_{21} f_{1}+a_{22} f_{2} \\
& \cdot \\
& \cdot \\
& \cdot \\
& u_{n}=a_{n 1} f_{1}+a_{n 2} f_{2}+\cdots+a_{n n-1} f_{n-1}+a_{n n} f_{n}
\end{aligned}
$$

where the $a_{i j}$ are determined by the orthogonality and normalization condition

$$
\begin{aligned}
\left(u_{i}, u_{j}\right)=\delta_{i j} & =1 ; i=j \quad i, j=1,2, \cdots, n . \\
& =0 ; i \neq j
\end{aligned}
$$

Since in the continuous case the orthonormalization is with respect to the contour integral $\int_{c} f(x, y) u(x, y) d s$, the inner product is defined by

$$
\begin{gathered}
\left(f_{i}, u_{j}\right)=\sum_{k=1}^{N} w_{k} f_{i k} u_{j k} \quad N=35, w_{1}=w_{1,1} w_{2}=w_{1,2} \cdots w_{7}=w_{1,7}, \\
w_{8}=w_{2,1} \quad \cdots \quad w_{15}=w_{2,7} \\
\cdot \\
\cdot \\
\cdot \\
w_{35}=w_{5,7}
\end{gathered}
$$


where the weights $w_{k}$ are given by the integration formula. Here the components of the vectors $f_{i}$ are the values of the corresponding harmonic functions of the basic set $1, \operatorname{Re} z, \operatorname{Im} z, \operatorname{Re} z^{2} \cdots$, in the cases $a, b$; or of the set $u_{0}, \operatorname{Re} z, \cdots$, in case $c$, at the 35 points given by the integration rule.

The approximate solution $u(x, y)$ of the Dirichlet problem is given then by the sum

$$
u(x, y)=\sum_{i=1}^{n} d_{i} f_{i}(x, y)
$$

where

$$
d_{i}=\sum_{k=1}^{i}\left(U, u_{k}\right) a_{k i} \quad i=1,2, \ldots \quad \cdot, n .
$$

$U$ is a vector the components of which are given by the boundary values of the Dirichlet problem in the points defined by the integration rule; $f_{i}(x, y)$ is the harmonic function corresponding to the vector $f_{i}$.

$u(x, y)$ has the property that among all the possible approximations of the form

$$
\sum_{k=1}^{n} c_{k} f_{k}(x, y)
$$

the deviation from the exact solution $U_{e}(x, y)$ is least in the least square sense:

$$
\begin{aligned}
& \text { if } \Delta=U_{e}-\sum_{i=1}^{n} d_{i} f_{i}(x, y)=U_{e}-u \\
& \text { then }(\Delta, \Delta)=\left(U_{e}-u, U_{e}-u\right)=\text { minimum. }
\end{aligned}
$$

Nehari [1] has given the following estimate for the deviation in the region where the Dirichlet problem is defined

$$
\begin{aligned}
\left(U_{e}(x, y)-\sum_{i=1}^{n} d_{i} f_{i}(x, y)\right)^{2} \leqq(\Delta, \Delta) \cdot \mid \frac{1}{2 \pi^{2}} \int_{c} \frac{d S}{(X-x)^{2}+(Y-y)^{2}} & -\sum_{i=1}^{n} u_{i}{ }^{2}(x, y) \mid .
\end{aligned}
$$

3. Numerical results. For each of the three cases $a, b$, and $c$ the number $n$ of harmonic functions in the basic set was varied in cases $a$ and $b$ from 3 to 11 , in case $c$ from 4 to 12 . Due to the limitations in internal storage capacity it was not possible to go higher than 12. The functions were numbered in the following way

$f_{1}=1, f_{2}=\operatorname{Re} z=x, f_{3}=\operatorname{Im} z=y, f_{4}=\operatorname{Re} z^{2}=x^{2}-y^{2}, f_{5}=\operatorname{Im} z^{2}=2 x y$

$f_{6}=\operatorname{Re} z^{3}, f_{7}=\operatorname{Im} z^{3}, f_{8}=\operatorname{Re} z^{4}, f_{9}=\operatorname{Im} z^{4}, f_{10}=\operatorname{Re} z^{5}, f_{11}=\operatorname{Im} z^{5}$.

The variation of $n$ was done in order to observe the convergence of the approximations to the exact solution of the Dirichlet problem. Due to the limited accuracy of the numerical computations (the orthonormalizing code works with floating decimal numbers containing only 8 digits) it must be expected that the improvement of the approximation with increasing $n$ will level off until the addition of another 
harmonic function $f_{i}$ to the set will not improve the approximation at all. In the three cases this point has not yet been reached, as one can see from table 1 , in which the norm of the vector consisting of the deviations in the points given by the integration rule is listed.

TABLE 1. Norm of Error Vector $\Delta$ Harmonic Functions

$\begin{array}{lrrrrr}\text { Case } & f_{1}, f_{2}, f_{3} & f_{1} \text { to } f_{5} & f_{1} \text { to } f_{7} & f_{1} \text { to } f_{9} & f_{1} \text { to } f_{11} \\ a & 18.007 & 9.565 & 8.822 & 2.500 & 2.061 \\ b & .351 & .214 & .157 & .107 & .076 \\ c_{1}{ }^{*} & .176 & .100 & .094 & .081 & .057 \\ c_{2}{ }^{*} & .126 & .077 & .026 & .023 & .022 \\ c_{3}{ }^{*} & .219 & .158 & .149 & .115 & .113 \\ c_{4}{ }^{*} & & .099 & .059 & .038 & .037 \\ c_{5}{ }^{*} & & & & .086 & .078\end{array}$

* In these cases $u_{0}$ was used in addition to $f_{1}, \cdots, f_{n}$, and $c_{i}$ is the case where the boundary values are one on the side $i$ (see Figure 1 ) and zero on the rest of the boundary. One observes that in the case $a$ the norm decreases more rapidly between $n=7$ and 9. The norm for $n=8$ is 2.517 and shows that the decrease occurs if $R e z^{4}$ is added to the set. The explanation for this behavior is probably that the function $\operatorname{Re} z^{4}=x^{4}+y^{4}-6 x^{2} y^{2}$ with its changes of sign along the lines $x=$ $( \pm 1 \pm \sqrt{2}) y$ (all combinations of signs are possible) fits particularly well the prescribed boundary conditions.

In table 2 the maximum error is given for the points on the boundary defined by the integration rule.

\section{TABLE 2}

Maximum Error in the Boundary Points Defined by the Integration Rule

\begin{tabular}{crrrrrr}
$\begin{array}{c}\text { Number of } \\
\text { functions }\end{array}$ & 3 & \multicolumn{1}{c}{5} & 7 & \multicolumn{1}{c}{. } & \multicolumn{1}{c}{$\begin{array}{c}\text { Max. bdry } \\
\text { value }\end{array}$} \\
Case & & & & & & \\
$a$ & 11.832 & 5.739 & 4.825 & 1.480 & .988 & 12.915 \\
$b$ & .232 & .148 & .109 & -.087 & -.066 & .595 \\
$c_{1}{ }^{*}$ & -.146 & -.065 & -.078 & -.068 & -.052 & 1.0 \\
$c_{2}{ }^{*}$ & -.106 & -.055 & -.029 & -.031 & -.028 & 1.0 \\
$c_{3}{ }^{*}$ & -.154 & -.115 & -.095 & -.091 & -.095 & 1.0 \\
$c_{4}{ }^{*}$ & & -.077 & .032 & .033 & .034 & 1.0 \\
$c_{5}{ }^{*}$ & & & & .070 & .079 & 1.0
\end{tabular}

From this table one sees that with eleven vectors the maximum relative error in cases $a$ and $b$ is approximately $10 \%$ on the boundary. (Since the deviation has been evaluated only in a finite number of points it is of course possible that this error may be in some points somewhat larger.) In cases $c_{i}$ the relative error is in most cases smaller than $10 \%$ due to the use of an additional harmonic function $u_{0}$ in the basic set. One remarks also that the maximum relative error for the first few approximations to the harmonic measures is much smaller than the error in the 
cases $a$ and $b$. This is due to the use of the corresponding harmonic measure $u_{0}$ for the circumscribed circle which gives a fairly good first approximation. From these observations one can conclude that from the numerical point of view the basic set of harmonic functions which has to be orthonormalized should not be chosen arbitrarily. The present computations show that a sophisticated choice of these functions creates considerably better approximations for the same number of orthonormal functions.

The Nehari estimates for the deviations in the point $x=y=0$ are given for the cases $a$ and $b$ in table 3 .

TABle 3. Deviations at $x=y=0$ and Nehari Estimates.

\begin{tabular}{lccccc}
\multicolumn{1}{l}{ Harmonic functions } & $f_{1}$ to $f_{3}$ & $f_{1}$ to $f_{5}$ & $f_{1}$ to $f_{7}$ & $f_{1}$ to $f_{9}$ & $f_{1}$ to $f_{11}$ \\
Case & & & & & \\
\multicolumn{1}{l}{$\begin{array}{l}\text { N Nehari estimate } \\
\text { actual error (abs.) }\end{array}$} & 5.19 & 2.74 & 2.41 & .643 & .528 \\
$b$ Nehari estimate & .855 & .495 & .646 & .0163 & .0133 \\
$\quad$ actual error (abs.) & .1010 & .0612 & .0428 & .0276 & .0195 \\
& .0099 & .0015 & .0050 & .0017 & .0002
\end{tabular}

From this table one sees that the estimate is still considerably larger than the actual error and that it converges slower than the actual error with increasing number of harmonic functions. If one compares, however, this estimate to the maximum error on the boundary given by table 2 it is evident that the Nehari estimate gives better error bounds than the one which can be derived from the maximum modulus principle for harmonic functions. In table 4 the Nehari estimates for the cases $a$ and $b$ are given for a number of points in the interior of the pentagon and for 11 harmonic polynomials.

\section{TABLE 4}

$\begin{array}{llllllll}\text { Case } \quad(y=0) & x=-2 & x=-1 & x=0 & x=1 & x=2 & x=3 \\ a & \text { Nehari estimate } & 1.798 & .539 & .528 & .528 & .614 & 1.631 \\ & \text { actual error } & .052 & .094 & .0133 & .0105 & .0181 & .406 \\ b \quad \text { Nehari estimate } & .0663 & .0199 & .0195 & .0195 & .0226 & .0601 \\ & \text { actual error } & .0091 & .0023 & .0002 & .0021 & .0038 & .0086\end{array}$

Table 4 shows that the Nehari estimates become worse as the boundary of the region is approached if they are compared with the estimates given by the maximum modulus principle. With respect to the actual error, however, the Nehari estimates are further off near the middle of the region than near the boundary.

In table 5 the Nehari estimates for the harmonic measures of the sides in the case where 12 harmonic functions were used are given for $x=y=0$ together with the maximum error in the points of the boundary given by the integration rule.

In these cases a comparison to the exact error is no longer possible since the exact solution is not known. The values show however that the Nehari estimate gives a better bound for the error at the origin than the one suggested by the maximum error on the boundary. 
TABLE 5

Nehari Estimates at $x=y=0$ and Maximum Error for Harmonic Measures

$\begin{array}{cccccc}\text { Case } & C_{1} & C_{2} & C_{3} & C_{4} & C_{5} \\ \begin{array}{c}\text { Nehari estimate at } \\ \begin{array}{c}x=y=0 \\ \text { Maximum error }\end{array}\end{array} & .0146 & .00556 & .0239 & .00945 & .0201 \\ & -.0520 & -.0279 & -.0945 & .0335 & .0788\end{array}$

4. Concluding Remarks. The best approximation in each of the seven cases gave values which are exact within at most ten per cent for many points of the region. Similar computations by P. Davis and P. Rabinowitz [6] show that with the given limited accuracy of the code the addition of further harmonic polynomials beyond Im $z^{5}$ would probably no longer improve this result considerably due to the influence of the round-off errors. In order to improve the accuracy one probably has to use at least double precision in the computations.

The numerical examples given on the previous pages show that the Nehari estimate gives a considerably better bound for the error in the interior of the region than the maximum modulus principle. With respect to the actual error the Nehari estimate is, however, still off by a factor which varied in the examples from 5 to almost 100 . It may be that the situation improves if more harmonic polynomials are used with a double precision code, but this can be decided only by experiments using computing machines which have a larger high speed memory than the SEAC. Since the use of orthonormal functions for solving problems in potential theory seems particularly attractive if the solution has to be known only in certain points of the region it would be interesting to see how much the present results can be improved by using double precision codes on the faster large computers like the IBM 704 or Remington Rand 1103AF.

The author wishes to thank Dr. P. Davis for many valuable suggestions and G. Blakley, J. P. Menard, and B. Urban for performing the necessary computations.

Computation Center,

\section{U. W. Hochstrasser}

University of Kansas,

Lawrence, Kansas

1. Zeev Nehari, On the Numerical Solution of the Dirichlet Problem, Proceedings of the Conference on Differential Equations, Univ. of Maryland Book Store, College Park, Md., 1956, p. 157-178.

2. S. Bergman, The Kernel Function and Conformal Mapping, Mathematical Surveys No. 5 , Am. Math. Soc., New York, 1950.

3. L. E. PAYNe \& H. F. Weinberger, "New bounds in harmonic and biharmonic problems," Jn. Math. and Physics, v. 33, 1955.

4. Philip Davis \& Philip Rabinowitz, "A multiple purpose orthonormalizing code and its uses." J. Assn. Comp. Machinery, v. 1, 1954, p. 183-191.

5. ZDENĚx Kopal, Numerical Analysis, John Wiley and Sons, Inc., New York, 1955, p. 390-394.

6. P. Davis \& P. Rabinowitz, "Numerical experiments in potential theory using orthonormal functions," Jn. Wash. Acad. of Sciences, v. 46, 1956, p. 12-17.

7. S. Zaremba, "Sur le calcul numérique des fonctions demandées dans le problème de Dirichlet et le problème hydrodynamique," International de l'Académie des Science de Cracovie, Bulletin, 1909, p. 125-195. 